\title{
Supplementary Information for „Tropical and mid-latitude teleconnections interacting with the Indian summer monsoon rainfall: A Theory-Guided Causal Effect Network approach“"
}

Giorgia Di Capua ${ }^{1,2}$, Marlene Kretschmer ${ }^{1}$, Reik V. Donner ${ }^{1,3}$, Bart van den Hurk ${ }^{2,4}$, Ramesh Vellore ${ }^{5}$, Raghavan Krishnan ${ }^{5}$, and Dim Coumou ${ }^{1,2}$

${ }^{1}$ Potsdam Institute for Climate Impact Research, Potsdam, Germany

${ }^{2}$ VU University of Amsterdam, Institute for Environmental Studies, Amsterdam, Netherlands

${ }^{3}$ Magdeburg-Stendal University of Applied Sciences, Magdeburg, Germany

$15{ }^{4}$ Deltares, Delft, Netherlands

${ }^{5}$ Indian Institute for Tropical Meteorology, Pune, India

Correspondence to: Giorgia Di Capua (dicapua@pik-potsdam.com)

PCMCI algorithm and numerical example

The PC-step has one main free parameter $\alpha$, which is the significance threshold chosen to retain or discard a certain parent

25 after calculating the partial correlation. In the latest version of the algorithm (TIGRAMITE 3.0, 
https://github.com/jakobrunge/tigramite), this value can either be set as a single value, e.g. $\alpha=0.05$, or as a set of values (the default set is $\alpha=\{0.05,0.1,0.2,0.3,0.4,0.5\}$ ). In the second case, the best $\alpha$ value is chosen by applying the Akaike information criterion (AIC), which compares the parents obtained with each element of $\alpha$.

The PC-step starts by first identifying the set of actors that we want to analyse. As an example we assume that our set of actors

30 is composed by 7 univariate time series which we hypothesize to share causal relationships. This set is referred to as the initial parents and is defined as follows:

$$
P=\{A, B, C, D, E, F, G\}
$$

Each letter in $P$ represents a univariate time series, which in our case must be given in the form of detrended anomalies. The PC algorithm first calculates plain correlations between the first element (at lag 0 ) and each of the remaining elements in $P$ at 35 a certain lag $\tau$ (here $\tau=1,2$ ). Let us assume that $A_{\tau=0}$ is found to be significantly correlated with 3 other actors, which will form the set of initial parents for $A_{\tau=0}$ (note that actors in $\widehat{P_{0}}$ are ordered by the strength of the correlation):

$$
\widehat{P_{0}}\left(A_{\tau=0}\right)=\left\{C_{\tau=-1}, D_{\tau=-2}, E_{\tau=-1}, G_{\tau=-1}\right\}
$$

For each element in $\hat{P}\left(A_{\tau=0}\right)$, partial correlations are calculated conditioning on the first strongest correlation. The partial correlation between the variables $x$ and $y$ conditioned on variable $z$ is calculated by first performing linear regressions of $x$ on

$40 \quad z$ and of $y$ on $z$ and then calculating the correlation between the residuals:

$$
\rho=\rho(\mathrm{x}, \mathrm{y} \mid \mathrm{z})=\rho(\operatorname{Res}(\mathrm{x}), \operatorname{Res}(\mathrm{y}))
$$

If the partial correlation between $x$ and $y$ is still significant at a certain significance level $\alpha, x$ and $y$ are said to be conditionally dependent given variable $z$, i.e. the correlation between $x$ and $y$ cannot be (exclusively) explained by the influence of variable $z$. When the opposite happens, the link is thus spurious and therefore filtered out and $x$ and $y$ are conditionally independent.

45 Following our example, the algorithm would proceed as follows:

$$
\begin{gathered}
\rho=\rho\left(A_{\tau=0}, C_{\tau=-1} \mid D_{\tau=-2}\right)=0.3, \mathrm{p}<0.05 \\
\rho=\rho\left(A_{\tau=0}, D_{\tau=-2} \mid C_{\tau=-1}\right)=-0.23, \mathrm{p}<0.05 \\
\rho=\rho\left(A_{\tau=0}, E_{\tau=-1} \mid C_{\tau=-1}\right)=0.35, \mathrm{p}<0.05 \\
\rho=\rho\left(A_{\tau=0}, G_{\tau=-1} \mid C_{\tau=-1}\right)=-0.18, \mathrm{p}>0.05
\end{gathered}
$$

50 In each step, the algorithm calculates the partial correlation between $A_{\tau=0}$ and each of the remaining elements of $\hat{P}\left(A_{\tau=0}\right)$ conditional on the first strongest parents $\left(C_{\tau=-1}\right.$ for all parents except for $C_{\tau=-1}$ itself where the second strongest, $D_{\tau=-2}$, is used). After this step, the set of parents is reduced to

$$
\widehat{P_{1}}\left(A_{\tau=0}\right)=\left\{C_{\tau=-1}, D_{\tau=-2}, E_{\tau=-1}\right\}
$$

Now, the algorithm tests the remaining parents conditioning on a subset of two variables, again starting from the strongest:

$$
\begin{gathered}
\rho=\rho\left(A_{\tau=0}, C_{\tau=-1} \mid D_{\tau=-2}, E_{\tau=-1}\right)=0.29, \mathrm{p}<0.05 \\
\rho=\rho\left(A_{\tau=0}, D_{\tau=-2} \mid C_{\tau=-1}, E_{\tau=-1}\right)=-0.17, \mathrm{p}>0.05 \\
\rho=\rho\left(A_{\tau=0}, E_{\tau=-1} \mid C_{\tau=-1}, D_{\tau=-2}\right)=0.27, \mathrm{p}<0.05
\end{gathered}
$$

The resulting set of parents will now be: 


$$
\widehat{P_{2}}\left(A_{\tau=0}\right)=\left\{C_{\tau=-1}, E_{\tau=-1}\right\}
$$

60 In the next step, the algorithm would test a combination of three variables. In our example, this is no longer possible because the set of parents now contains only two variables. When the number of parents becomes smaller or equal to the number of conditions that should be tested, the algorithm converges for $A$ and starts to test the parents of $B$, following exactly the same process. Let us assume that after testing all the actors in $P$, we find three sets of parents:

$$
\begin{aligned}
& \widehat{P_{2}}\left(A_{\tau=0}\right)=\left\{C_{\tau=-1}, E_{\tau=-1}\right\} \\
& \widehat{P_{3}}\left(C_{\tau=0}\right)=\left\{A_{\tau=-1}, D_{\tau=-1}\right\} \\
& \widehat{P_{2}}\left(E_{\tau=0}\right)=\left\{B_{\tau=-1}, F_{\tau=-1}\right\}
\end{aligned}
$$

The selected sets of parents then enter the second step of PCMCI. The PC-step has one main free parameter $\alpha$, which is the significance threshold chosen to retain or discard a certain parent after calculating the partial correlation.

In the MCI-step, the partial correlation between an actor and its set of parents is calculated again but conditioning also on the

70 sets of parents of the parents of the actor we are interested in. Following our example:

$$
\begin{gathered}
\rho=\rho\left(A_{\tau=0}, C_{\tau=-1} \mid A_{\tau=-2}, D_{\tau=-2}\right)=0.26, \mathrm{p}<0.05 \\
\rho=\rho\left(A_{\tau=0}, E_{\tau=-1} \mid B_{\tau=-2}, F_{\tau=-2}\right)=0.22, \mathrm{p}<0.05
\end{gathered}
$$

After this final test, both parents pass the MCI test and will then form the final set of parents for $A_{\tau=0}$. When a set of different $\alpha$ is used, the parents with the highest AIC score are used as conditions when calculating partial correlations in the MCI-step

75 (Runge et al., 2017). 


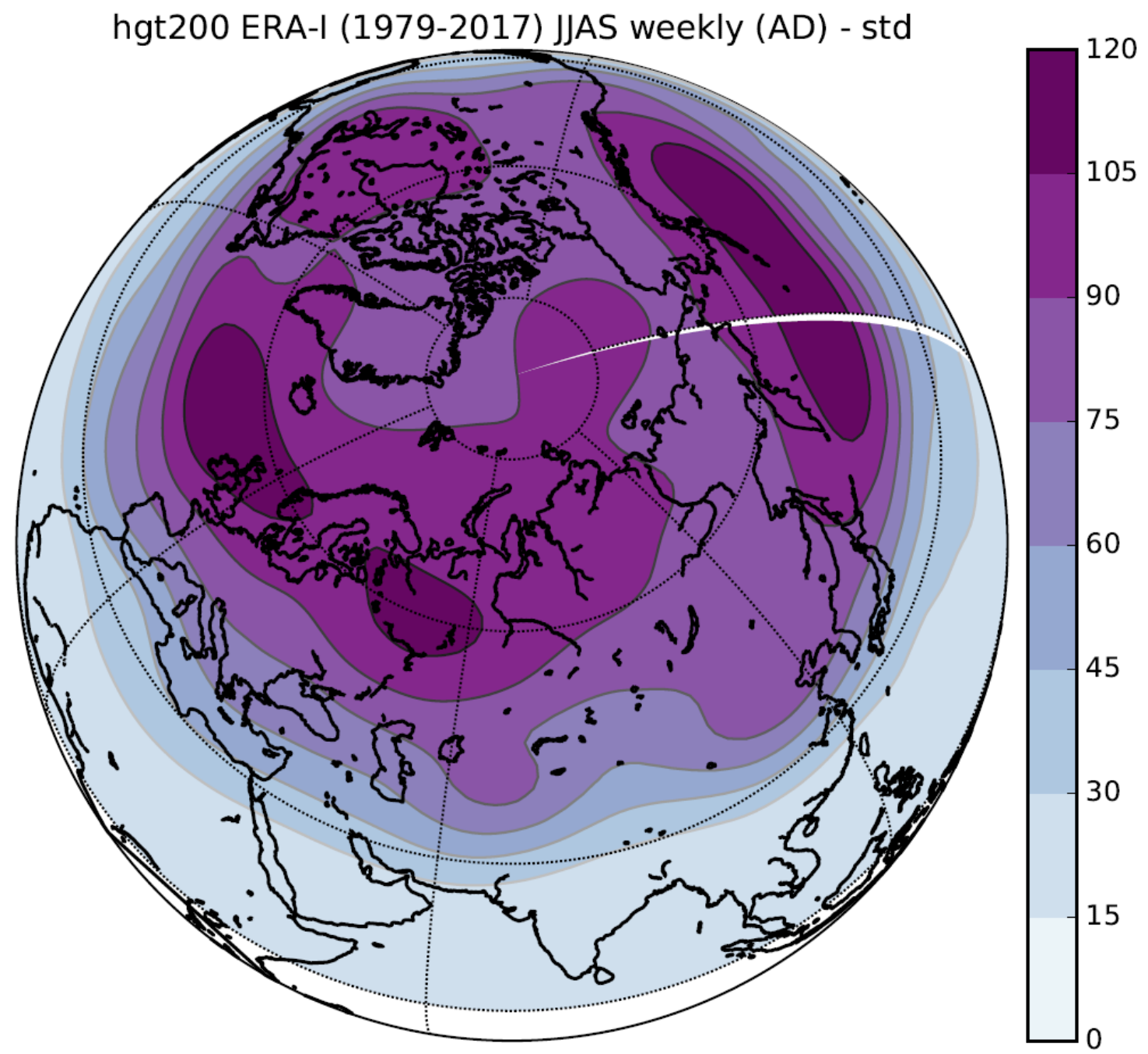

Figure S1. Standard deviation of weekly JJAS Z200 in the Northern Hemisphere for the period 1979-2017. 

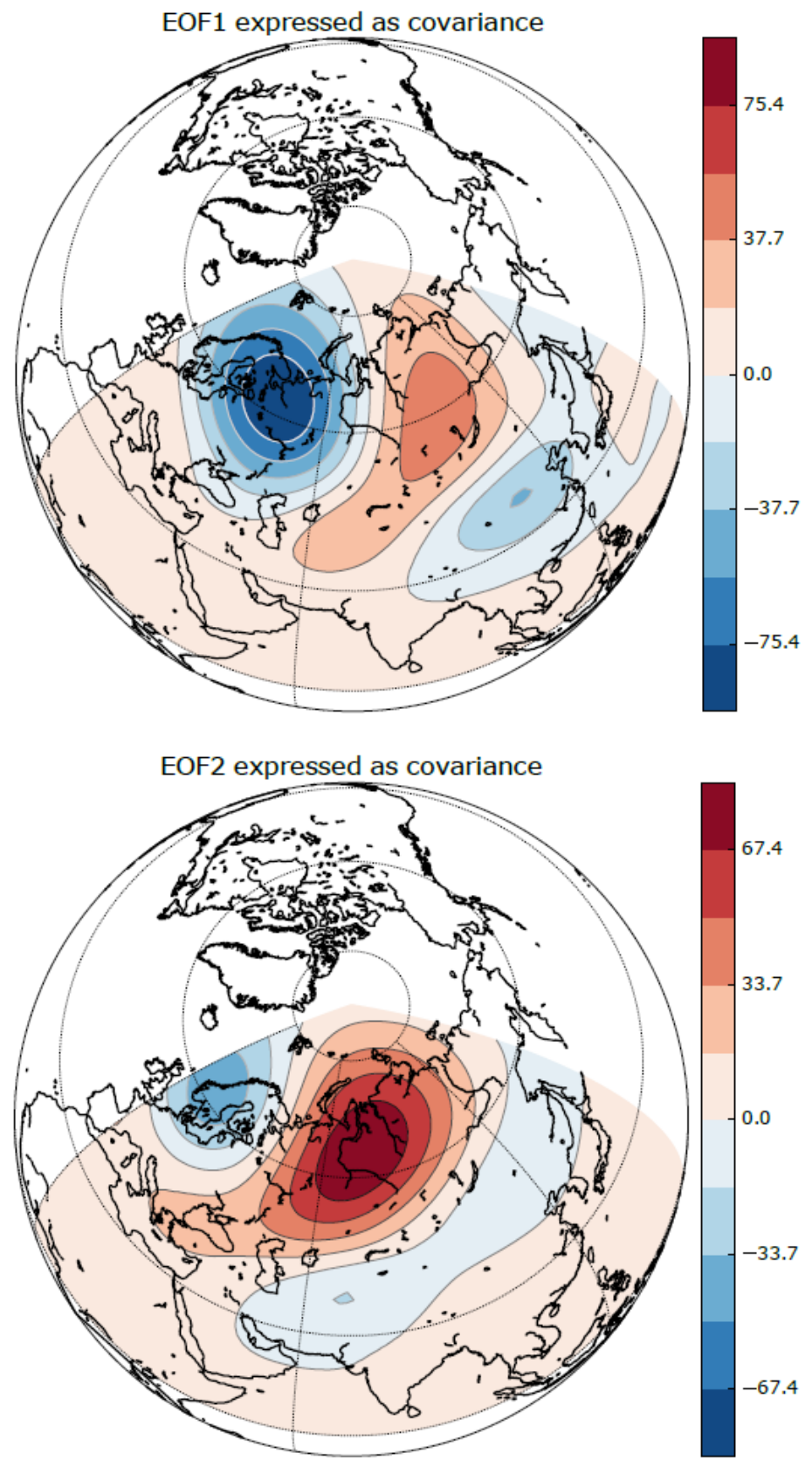

80

Figure S2. Standard deviation of weekly JJAS Z200 in the Northern Hemisphere for the period 1979-2016. 


\section{Z-L1}

Z-L2

CGTI

\section{MTrain}

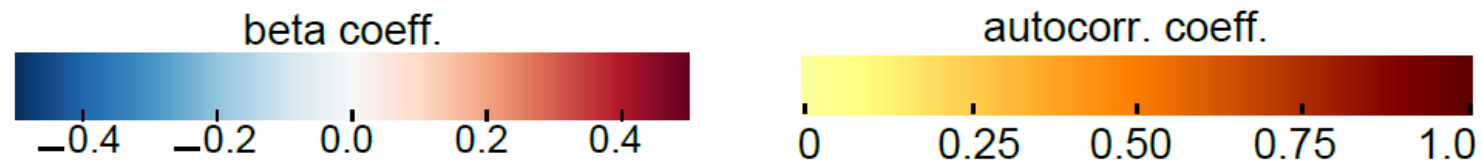

Figure S3. CEN built with weekly CGTI, MT rainfall L1 and L2 (as defined in Figure 5a in the main manuscript). MT rainfall is taken from Pai et al. dataset for the period 1979-2017. 


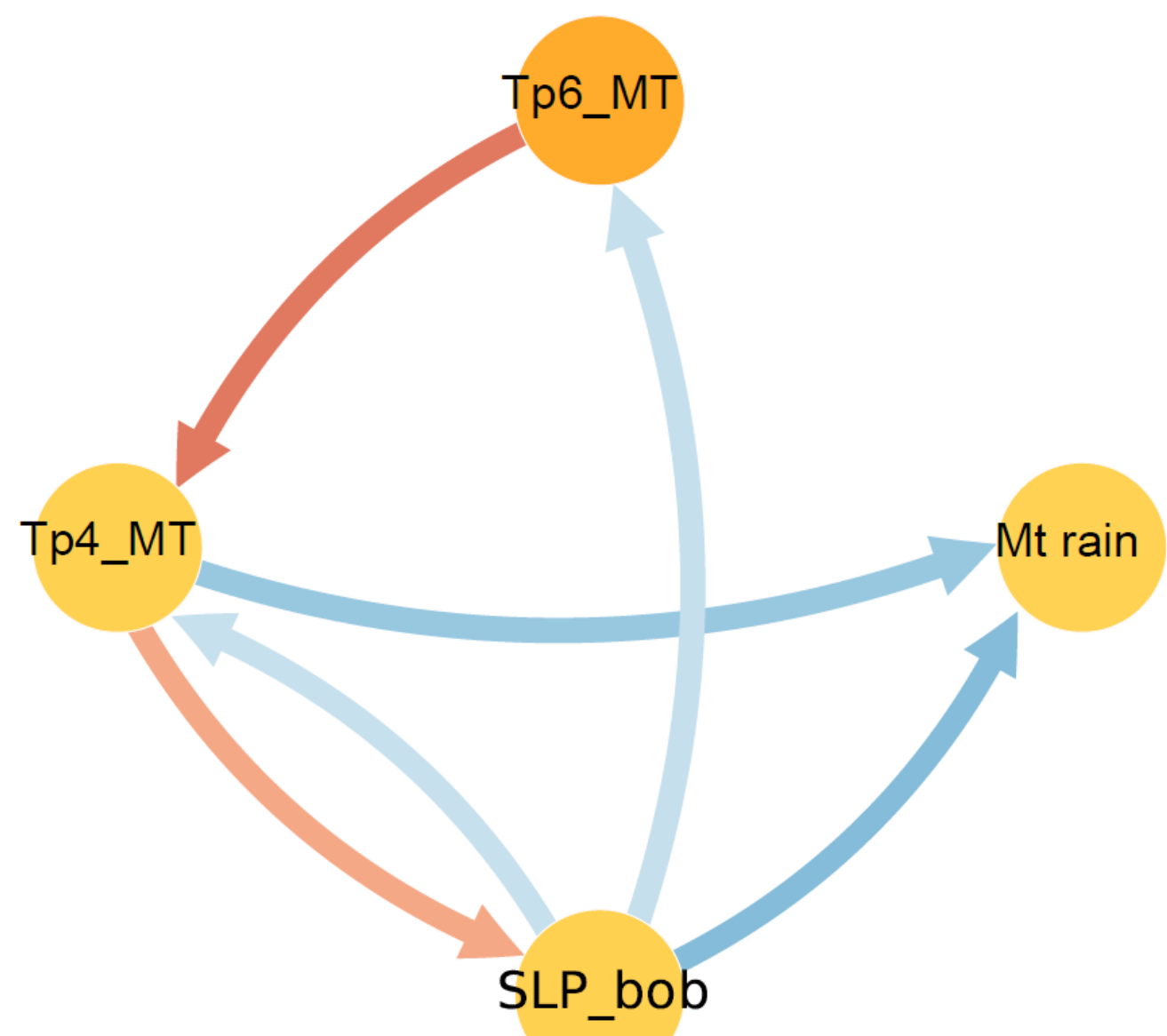

beta coeff.

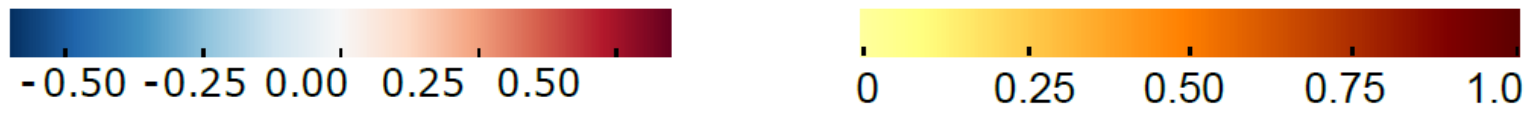

Figure S4. CEN built with MT rainfall from the CPC-NCEP dataset, SLP_bob, Tp4_MT, Tp6_MT for the 1979-2016 period. 
(a) JJAS climatology for CPC-Rainfall ( $\mathrm{mm}$ day-1)

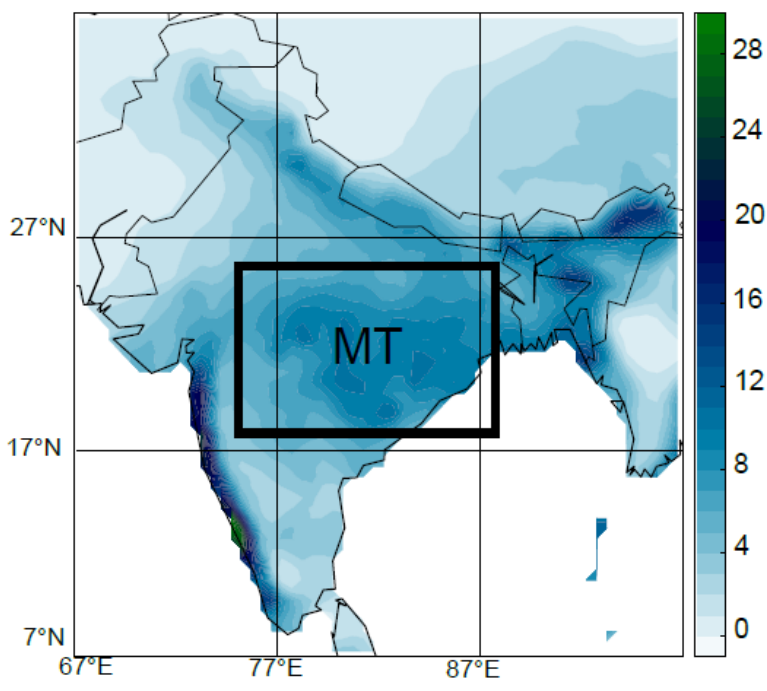

(b) weekly stand. dev. for CPC-Rainfall $\left(\mathrm{mm} \mathrm{day}^{-1}\right)$

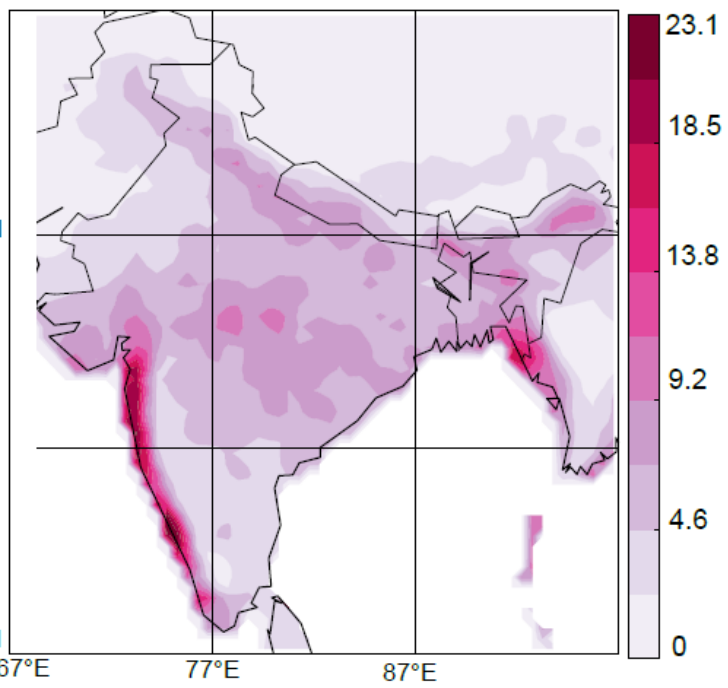

(c) JJAS weekly anomalies for CPC-Rainfall - 1979-2016

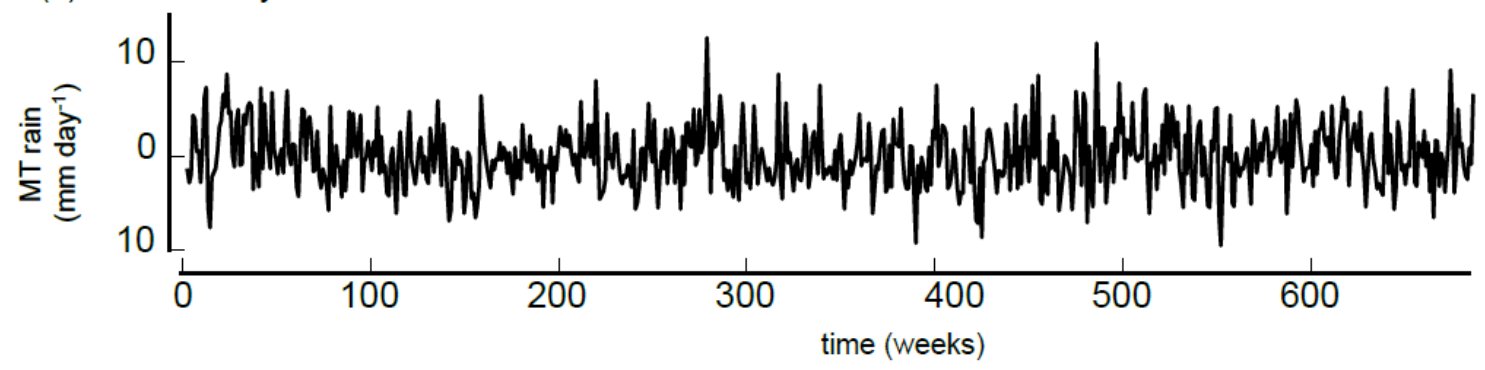

90 Figure S5. Rainfall climatology over India. Panel (a): JJAS rainfall climatology over the 1979-2016 period from the CPC-NCEP dataset. The black box identifies the MT region. Panel (b): standard deviation for weekly JJAS rainfall over the 1979-2016 period from the CPCNCEP dataset. Panel (c): time series of weekly MT rainfall over the period 1979-2016; each year contains 18 weeks, with the first week starting on the $27^{\text {th }}$ of May. 
(a) EOF1 of weekly Z200 (JJAS)

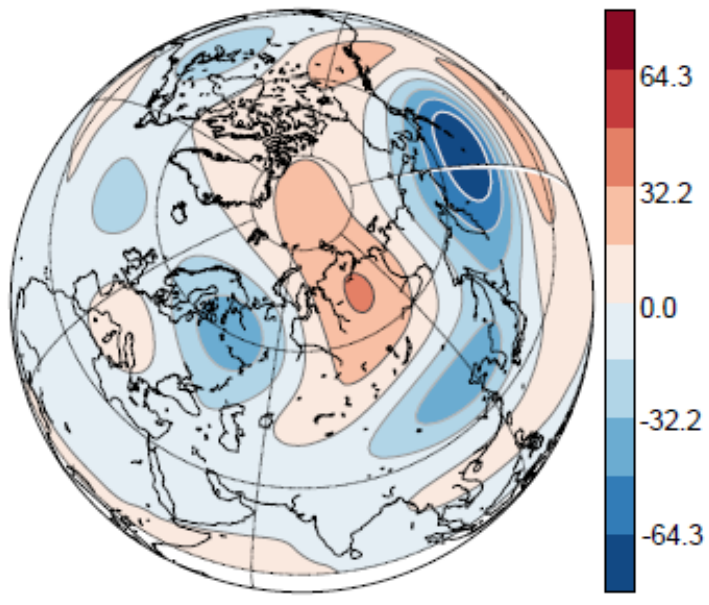

(b) EOF2 of weekly Z200 (JJAS)

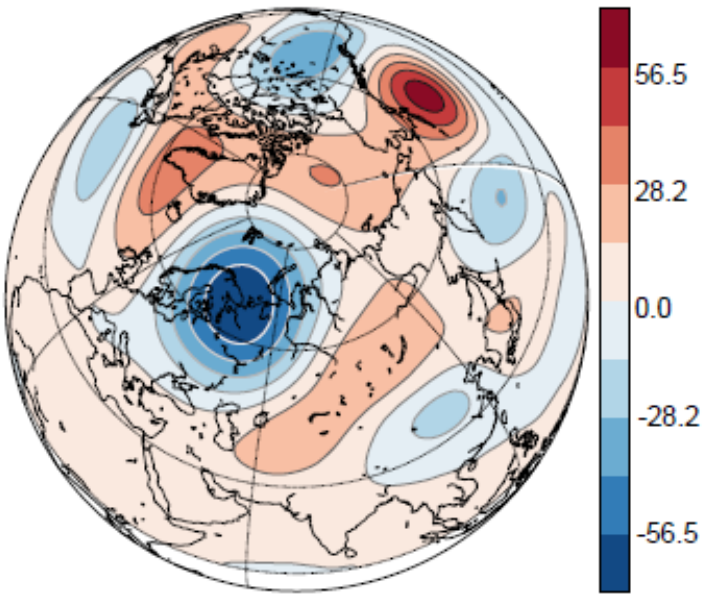

(c) Weekly correlation of Z200 with MTrain (d) Weekly correlation of Z200 with CGTI

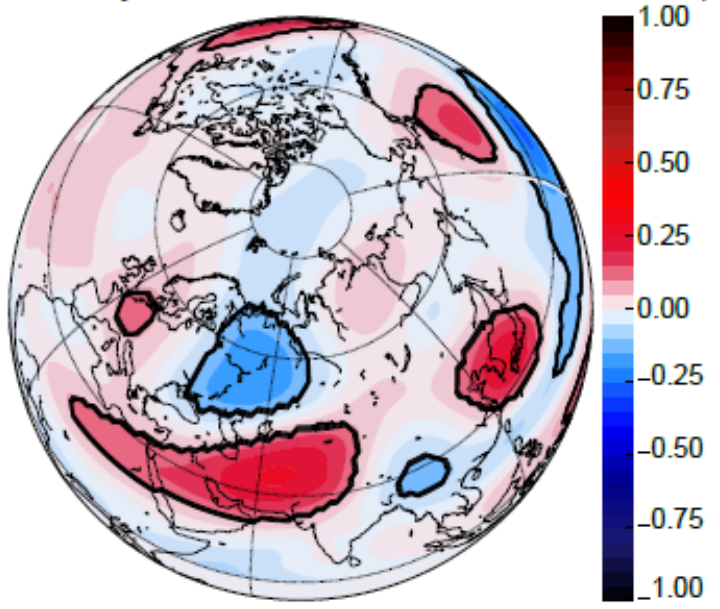

(e) Temperature anomalies (K)

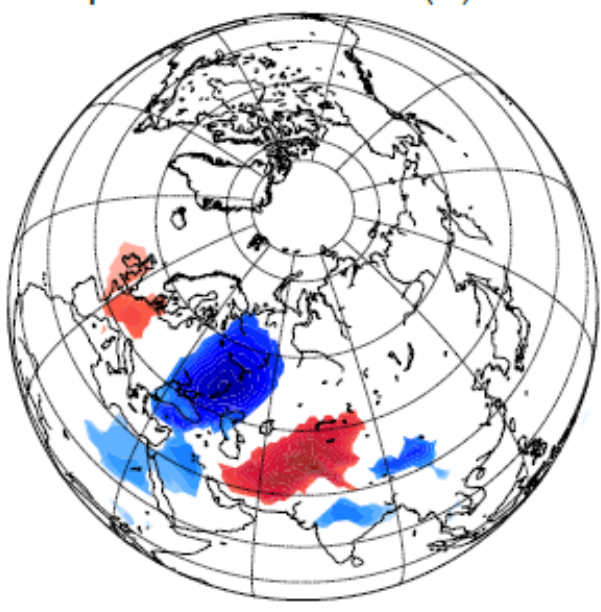

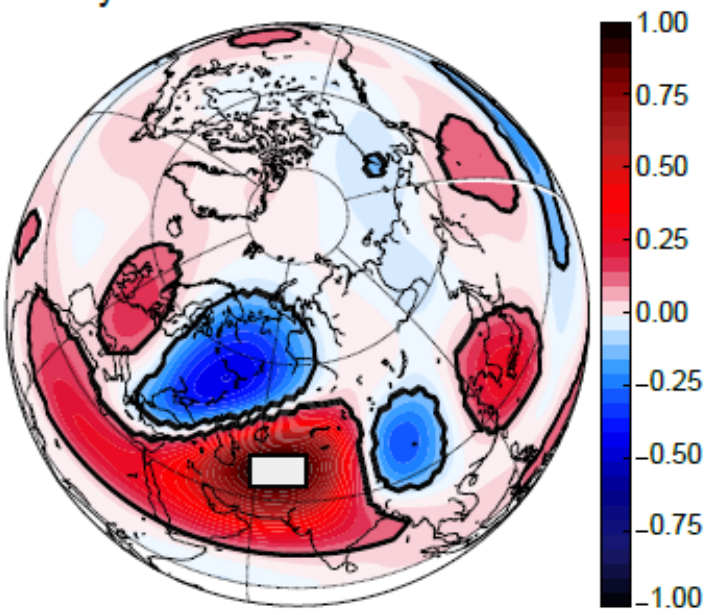

(f) Precipitation anomalies $\left(\mathrm{mm}\right.$ day $\left.^{-1}\right)$

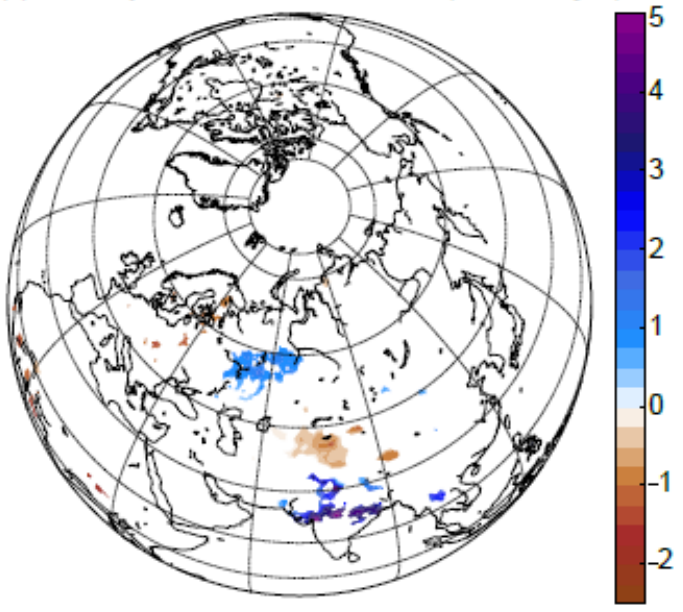


95 Figure S6. Mid-latitude variability associated with ISM. Panel (a) and (b): EOF1 and EOF2 for the JJAS weekly Z200 field in the northern mid-latitudes for the period 1979-2016. Panel (c): correlation between weekly MT rainfall and Z200 (lag = -1 week) for the period 19792016. Panel (d): the CGTI region (white box) and the correlation between CGTI and Z200 (lag = 0), which forms the circumglobal teleconnection pattern for the period 1979-2016. Panel (e): Temperature anomalies over the Northern Hemisphere during weeks with CGTI $>1$ std of CGTI minus weeks with CGTI < -1 std of CGTI for the period 1979-2016. Panel (f): as panel (e) but for rainfall anomalies. In 100 panels c,d,e,f only correlation coefficients and anomalies with $p<0.05$ are shown.

(a)

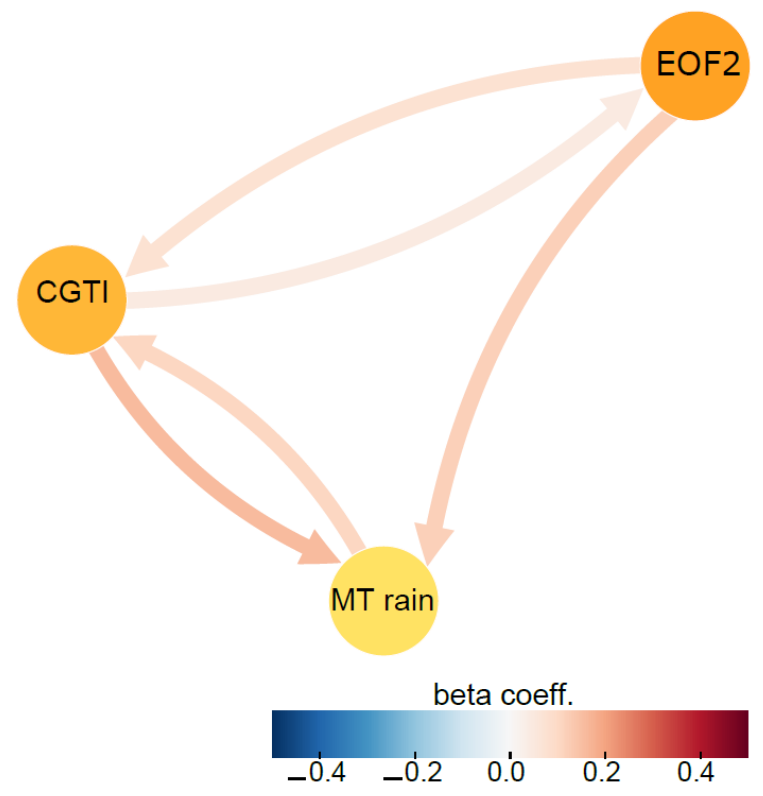

(b)

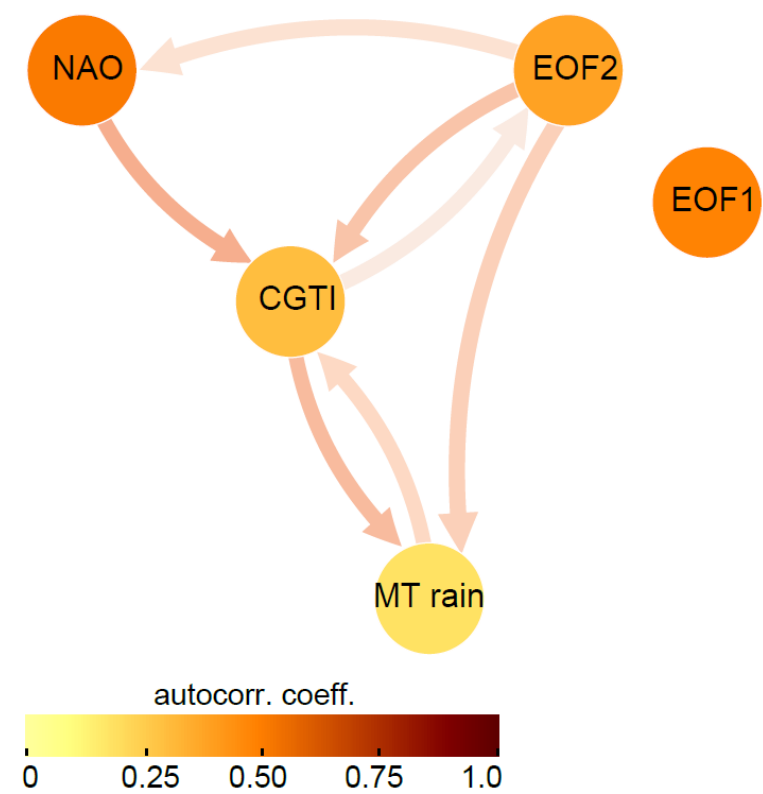

Figure S7. Causal mid-latitude interactions of the ISM. Panel (a): Causal Effect Network (CEN) built with CGTI, the PC of EOF2 and MT rainfall from CPC-NCEP dataset for the period 1979-2016. Panel (b): as panel (a) but with the addition of the EOF1 and NAO. 


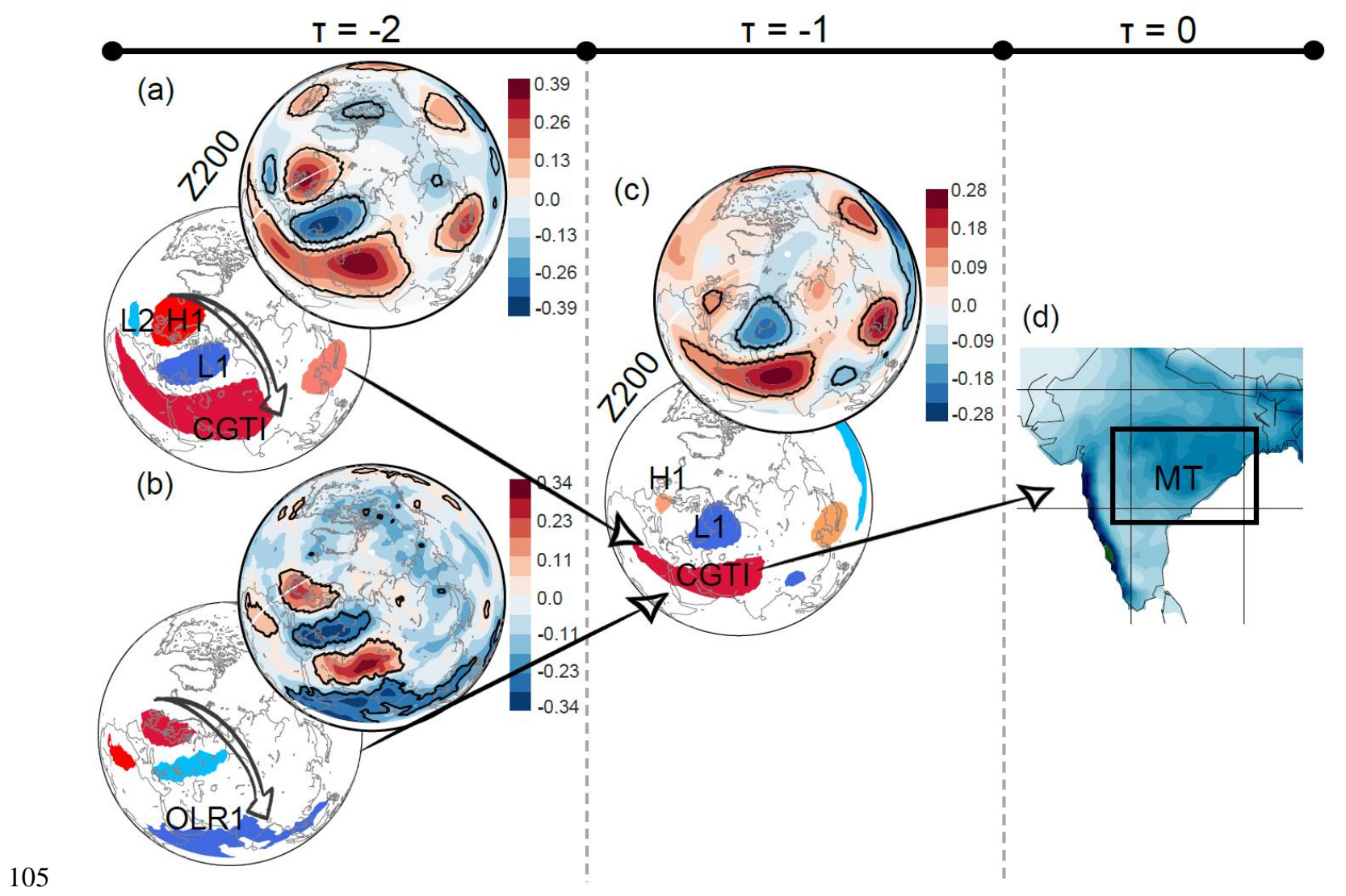

Figure S8. . Mid-latitude causal precursors of ISM. Panel (a): correlation of CGTI with Z200 at 1-week lead time (top panel), and the causal precursors of CGTI identified via RG-CPD (bottom panel) for the period 1979-2016. Panel (b): as for panel (a) but for OLR fields. Panel (c): correlation map for weekly MT rainfall and Z200 field at 1-week lead-time (top panel) and the causal precursors identified via RG-CPD (bottom panel) for the period 1979-2016. Panel (d): ISM rainfall over the MT region from the CPC-NCEP dataset for the period 110 1979-2016. 


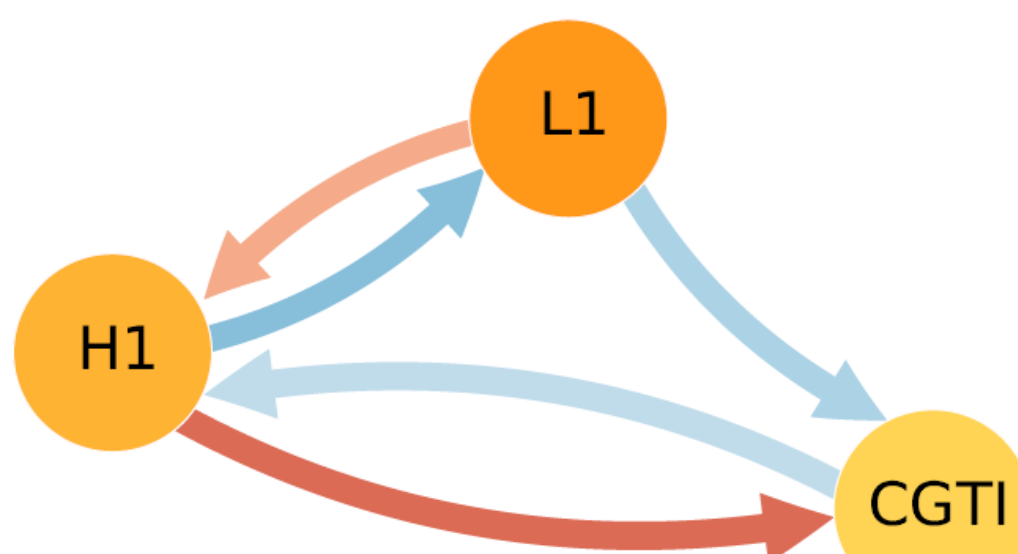

$M T$ rain

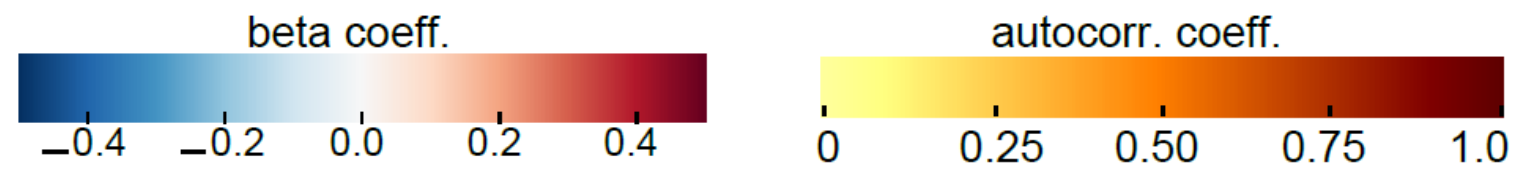

Figure S9. Mid-latitude wave train. CEN built with the MT from the CPC-NCEP dataset rainfall for the period 1979-2016, CGTI, L1 and H1 (as identified in Figure 5a). 
(a)

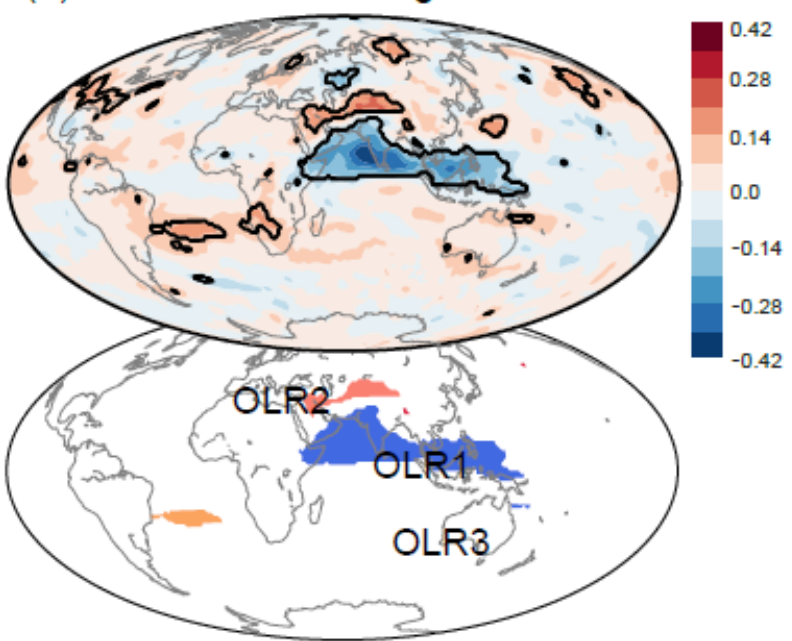

(c)

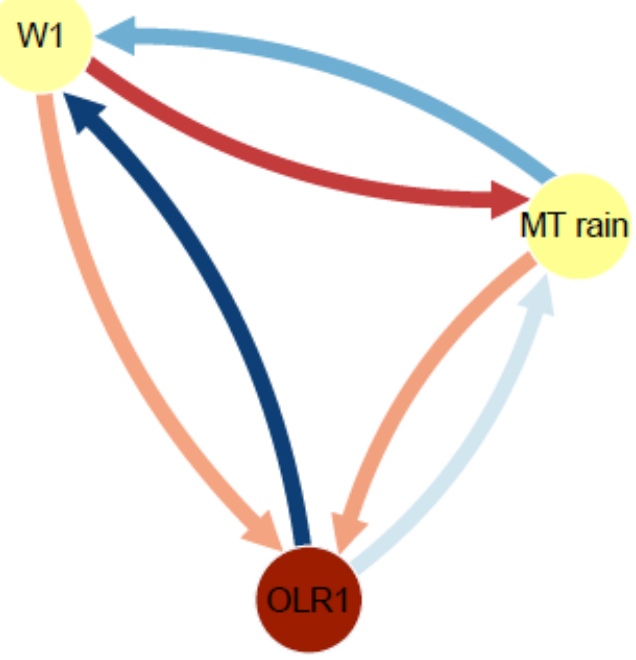

coeff.

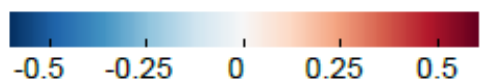

(b) $\quad \mathrm{W}, \mathrm{Lag}=-1$

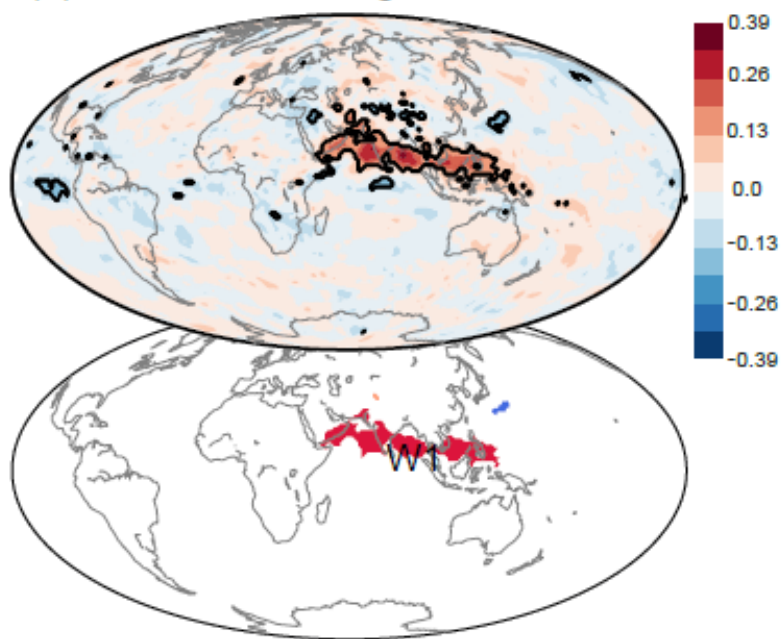

(d)

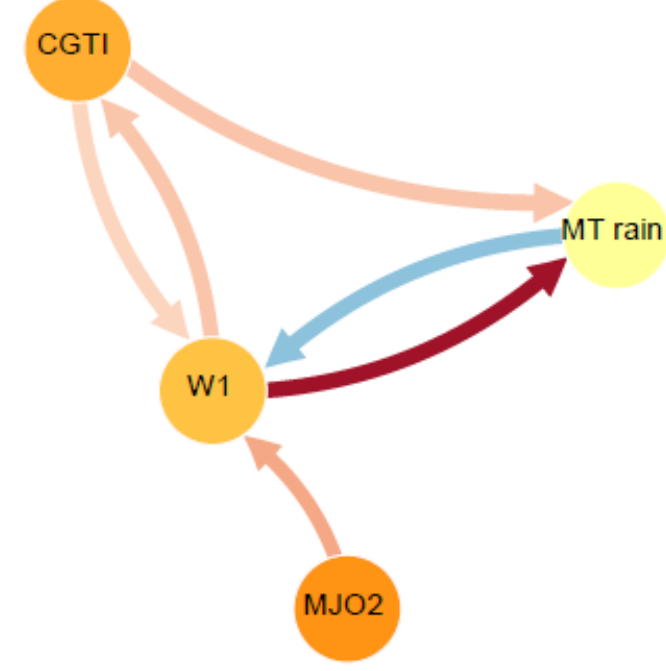

autocorr. coeff.

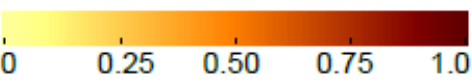

Figure S10. Tropical causal interactions of ISM. Panel (a) shows the correlation map for weekly MT rainfall from the CPC-NCEP dataset for the period 1979-2016 and the global OLR field at 1-week lead-time (top panel) and the causal precursors identified via RG- 
CPD (bottom panel). Panels (b): as for panel (a) but for W fields. Panel (c) and (d) show the CEN build with W1, OLR1 and MT rainfall and MT rainfall, W1, CGTI and MJO respectively.
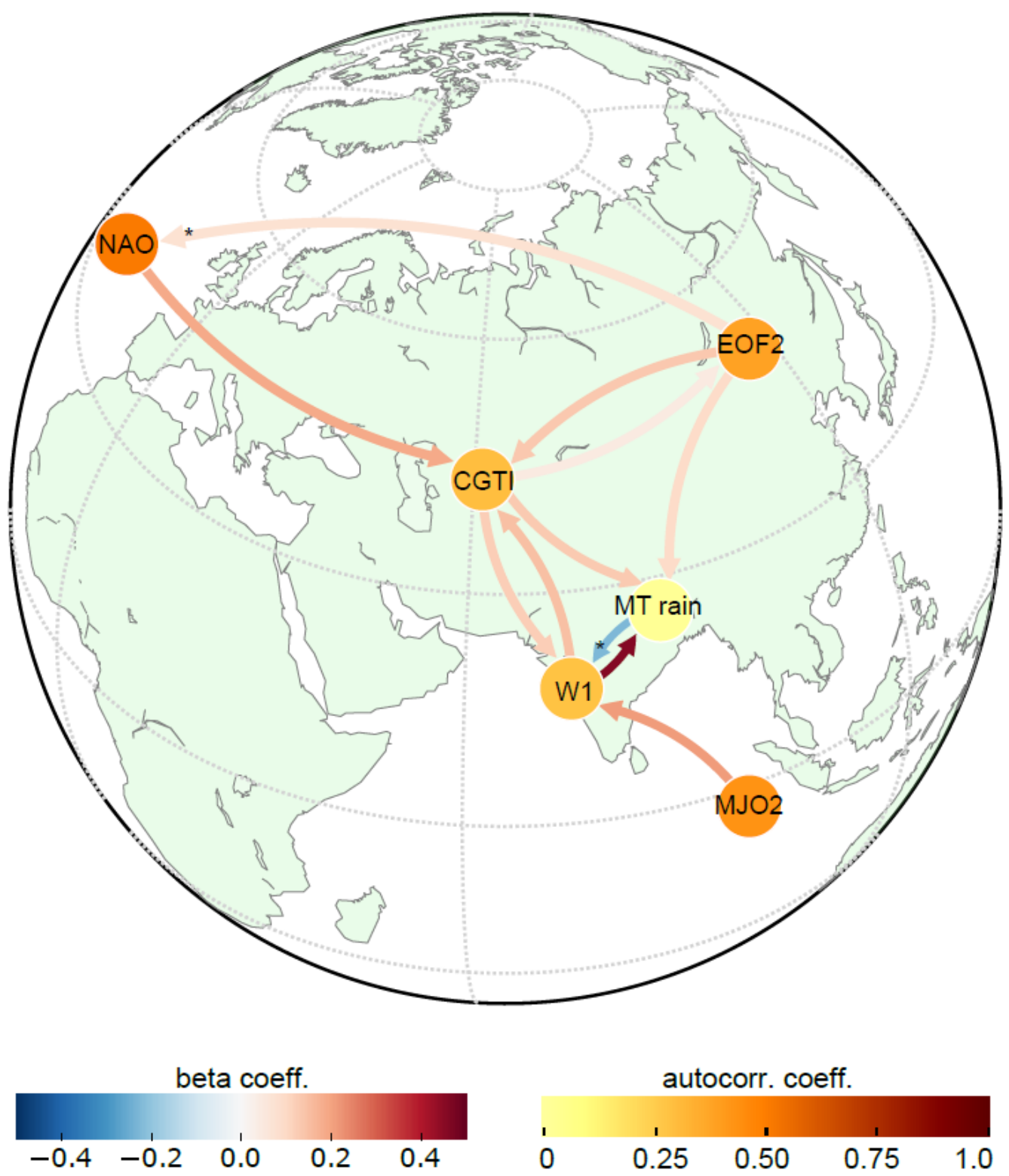

Figure S11. Combined mid-latitude and tropical causal interactions of ISM. CEN built with W1, MJO2, MT rainfall from the CPCNCEP dataset for the period 1979-2016, NAO, CGTI and EOF2. 


\begin{tabular}{|c|c|}
\hline Link & Link strength (CE) \\
\hline$\beta_{\mathrm{W} 1 \rightarrow \mathrm{MT}}$ & 0.53 \\
\hline$\beta_{\mathrm{MJO2} \rightarrow \mathrm{W} 1}$ & 0.29 \\
\hline$\beta_{\mathrm{CGTI} \rightarrow \mathrm{MT}}$ & 0.19 \\
\hline$\beta_{\mathrm{W} 1 \rightarrow \mathrm{MJO} 2}$ & -0.36 \\
\hline$\beta_{\mathrm{MT} \rightarrow \mathrm{W} 1}$ & -0.23 \\
\hline$\beta_{\mathrm{EOF} 2 \rightarrow \mathrm{NAO}}$ & 0.08 \\
\hline$\beta_{\mathrm{NAO} \rightarrow \mathrm{CGTI}}$ & 0.17 \\
\hline$\beta_{\mathrm{W} 1 \rightarrow \mathrm{CGTI}}$ & 0.17 \\
\hline$\beta_{\mathrm{EOF} 2 \rightarrow \mathrm{CGTI}}$ & 0.11 \\
\hline$\beta_{\mathrm{CGTI} \rightarrow \mathrm{EOF} 2}$ & 0.06 \\
\hline$\beta_{\mathrm{CGTI} \rightarrow \mathrm{W} 1}$ & 0.14 \\
\hline
\end{tabular}

Table S1. Causal effect (CE) values. CE values for links presented in Fig. 7 in the main text.

\begin{tabular}{|c|c|c|c|c|c|c|}
\hline & MT rainfall & NAO & CGTI & EOF2 & W1 & MJO2 \\
\hline ACE & 0.046 & 0.035 & 0.078 & 0.039 & 0.212 & 0.059 \\
\hline ACS & 0.142 & 0.016 & 0.092 & 0.012 & 0.133 & 0.072 \\
\hline
\end{tabular}

Table S2. Average causal effect (ACE) and average casual susceptibility (ACS). ACE and ACS for actors presented in Fig. 8 in the main text. 